\title{
André Thibault (éd.), Le français dans les Antilles: études linguistiques
}

\section{Chiara Brandolini}

\section{Q OpenEdition}

1 Journals

\section{Edizione digitale}

URL: http://journals.openedition.org/studifrancesi/1655

DOI: 10.4000/studifrancesi. 1655

ISSN: 2421-5856

\section{Editore}

Rosenberg \& Sellier

\section{Edizione cartacea}

Data di pubblicazione: 1 novembre 2014

Paginazione: 646-648

ISSN: 0039-2944

\section{Notizia bibliografica digitale}

Chiara Brandolini, «André Thibault (éd.), Le français dans les Antilles: études linguistiques », Studi

Francesi [Online], 174 (LVIII | III) | 2014, online dal 01 novembre 2014, consultato il 18 septembre 2020. URL : http://journals.openedition.org/studifrancesi/1655; DOI : https://doi.org/10.4000/studifrancesi. 1655

Questo documento è stato generato automaticamente il 18 settembre 2020.

\section{(c)}

Studi Francesi è distribuita con Licenza Creative Commons Attribuzione - Non commerciale - Non opere derivate 4.0 Internazionale. 


\title{
André Thibault (éd.), Le français dans les Antilles: études linguistiques
}

\author{
Chiara Brandolini
}

\section{NOTIZIA}

ANDRÉ THIBAULT (éd.), Le français dans les Antilles: études linguistiques, Paris, l'Harmattan, 2012 , pp. 426.

1 Buona parte dei contributi di questa raccolta, curata e diretta da André Thibault, è costituita da una selezione di comunicazioni presentate nel corso del convegno «Le français régional antillais: exploration et délimitation d'un concept», tenutasi nel 2010 presso l'Université Paris-Sorbonne. Nell'introduzione al volume, Thibault presenta la novità del tema trattato e delinea le motivazioni del suo recente esordio: la natura del francese praticato nelle Antille è stata spesso trascurata dalla linguistica non solo perché la variazione diatopica del francese in generale è diventata un campo di studi importante solo a partire dall'ultimo decennio del xx secolo, ma anche perché l'attenzione dei ricercatori si è sempre appuntata di preferenza sullo studio del creolo. Si è pertanto sempre cercato di spiegare la genesi del creolo partendo dal francese e dal galloromanzo: il curatore, invece, si fa promotore di una tendenza contraria che concepisce il creolo come una miniera di dati diatopici e diastratici utili per studiare la storia del francese orale, popolare e quotidiano, poiché la storia del creolo e del francese nelle Antille rappresenta le due facce della stessa medaglia. Secondo l'autore, un altro fattore che ha reso difficile l'emergere degli studi sulle particolarità del francese nelle Antille sarebbe legato all'insicurezza linguistica che fa sì che i parlanti mantengano l'illusione di evolvere tra due lingue ben distinte, il creolo e il francese libresco: questa concezione non lascia posto alla considerazione di una varietà endogena di francese, spesso stigmatizzata sia dai difensori del creolo, sia dai difensori del "bon usage". Sempre nell'introduzione, il curatore cerca di definire l'oggetto di studio in maniera ampia, vale a dire il francese che è praticato oralmente e in forma scritta nelle Antille, dalle origini ai nostri giorni, da tutti i gruppi socio-etnici 
tradizionalmente impiantati nel luogo, in tutte le circostanze della vita. Ben conscio del fatto che nel xx secolo il francese nelle Antille diventa sempre più diffuso e sviluppa delle varianti proprie grazie alla scolarizzazione e ai mass media metropolitani, il curatore delimita l'oggetto di studio all'emergenza della nuova varietà di francese delle Antille parlato dalla stragrande maggioranza della popolazione e la cui esistenza è dovuta alla non-trasmissione del creolo come lingua materna in contesto familiare: si tratta quindi di analizzare una lingua madre con caratteristiche proprie all'orale, e che non si presenta limitata alle funzioni di prestigio. In questo modo, si prendono le distanze dal francese coloniale $\mathrm{e}$ dal francese più purista che percepisce automaticamente come creolo tutto ciò che è lontano dallo standard. Thibault pone l'accento poi sulla difficoltà di descrivere la frontiera e le relazioni tra creolo e francese, sottolineando, tramite le nozioni di diglossia, di interletto, di contatto e di continuum, che l'unanimità dei ricercatori è ancora lontana dal trovare una conclusione univoca. In seguito, il curatore si preoccupa di fornire un elenco delle pubblicazioni sul francese regionale delle Antille che permette di riunire dati sulle particolarità fonetiche, morfosintattiche e lessicali di questa varietà.

2 L'opera è strutturata in cinque sezioni: «Histoire et étymologie»; «Les diatopismes antillais dans le discours littéraire»; «Phonétique»; «Le français en Haïti»; «Lexicographie». La prima sezione fornisce nuovi dati su numerosi particolarismi lessicali tramite tre articoli: Annegret BoLléE, in Étymologies creoles. Contributions du Dictionnaire étymologique des créoles français d'Amérique (DECA) à l'histoire du vocabulaire régional antillais, presenta alcuni articoli del suo futuro dizionario dividendoli in termini di origine non francese e in "survivances", cioè parole cadute in disuso nel francese di Francia o di origine regionale o dialettale. Nello specifico, presenta articoli del dizionario che concernono parole di origine africana e amerindiana e, nella sezione "par les chemins de la mer", termini marinari o coloniali che sono stati presi a prestito dalle lingue di contatto e trasportati da una colonia all'altra. Il contributo di Jean-Paul CHAUVEAU, Des régionalismes de France dans le créole de Marie-Galante, cerca di caratterizzare il francese dei fondatori della colonia di Marie-Galante studiando i regionalismi del francese di Francia, in particolare quelli di origine normanna, che si sono trapiantati nei Caraibi così profondamente da essere stati adottati dai creoli. Egli arriva così a fornire nuovi dati sul francese parlato in epoca classica negli ambienti popolari francesi, evidenziando le aree di partenza degli apporti al francese e al creolo. L'ultimo articolo di questa sezione, La formation du français régional et des créoles antillais: l'apport du taïno di Silke JANSEN, si concentra sulle etimologie amerindiane, guardando più da vicino le parole per cui è stata proposta un'etimologia taïno, lingua parlata nelle grandi Antille prima dell'arrivo degli Europei e prima lingua amerindiana con cui questi entrano in contatto. Partendo da questi esempi, l'autrice fornisce nuovi dati caratterizzando in maniera più precisa l'apporto del taïno nella formazione del lessico francese e dei creoli a base francese dei Caraibi. Prima di tutto, descrive la situazione linguistica nelle Antille al momento dell'arrivo degli Spagnoli; in seguito, l'autrice propone una bozza dei sistemi fonologici delle lingue delle Antille precolombiane, dimostrando che non è per contatto diretto, ma tramite l'intermediazione dello spagnolo che le parole di taïno penetrano nel francese: questo contributo evidenzia che la conoscenza delle lingue precolombiane e l'analisi delle fonti spagnole possono fornire risposte molto importanti sul ruolo delle lingue indigene nella formazione del francese regionale e dei creoli nelle Antille. 
3 La seconda sezione presenta gli aspetti del francese delle Antille reperibili nel discorso letterario. Il primo articolo è Le français à la Dominique dans le discours romanesque: reconstruction linguistique ou construction discursive? in cui Inka WISSNER, dopo aver fotografato la situazione linguistica contemporanea di quest'isola, analizza il romanzo del francese Yves Violler Notre-Dame des Caraibes: grazie all'uso delle tecniche per l'analisi del discorso, egli cerca di stabilire lo statuto semiotico e stilistico degli antillanismi in esso contenuti. In appendice inserisce un lessico che riprende tutti gli antillanismi del romanzo presentati sotto forma di articoli redatti seguendo le tecniche della lessicografia differenziale francofona. Si passa poi al livello morfologico grazie all'articolo di Teodor ZANOAGA, Observations sur la formation des mots en français littéraire antillais: étude d'un corpus de littérature contemporaine: l'autore analizza i procedimenti di formazione (agglutinazione, aferesi, raddoppiamento, composizione, costruzioni seriali, télescopage e derivazioni varie) che caratterizzano i diatopismi rilevati in tre romanzi di un autore contemporaneo della Guadalupa, Ernest Pépin. L'ultimo articolo della sezione, Saint-John Perse à l'épreuve d'une traducion en portugais concerne la traduttologia, in particolare i problemi che Gabriel PINHEIRO, traduttore e autore del saggio, ha incontrato nella resa degli antillanismi polisemici che si concentrano nella prima parte dell'opera poetica del Premio Nobel Saint-John Perse. In questo caso, la traduzione si conferma come ricreazione del testo di partenza che si distingue per l'accumulazione di significati a tutti i livelli linguistici.

4 La sezione dedicata alla fonetica consta di due articoli. Quello di André THIBAULT, Les avatars du schwa colonial dans le créole des petites Antilles, mostra, grazie al principio della cronologia relativa che è utilizzato nella fonetica storica romanza, che il grafema <e> e il digramma <eu> dovevano obbligatoriamente rappresentare due suoni distinti nel francese dei coloni. Quest'articolo mette in luce l'importanza dei dati forniti dal creolo per la ricostruzione di quello che deve essere stato il francese parlato dai coloni. L'autore elenca poi i desiderata per completare tale ricerca: tra questi troviamo l'elaborazione di un trattato di fonetica storica per i creoli atlantici che potrebbe essere affiancato al futuro dizionario di Bollée. Dopo questo contributo diacronico, viene l'articolo di Elissa PUSTKA, Le caméléon dans la jungle sonore: variation du r en Guadeloupe, che studia in maniera approfondita la grande varietà di realizzazioni sonore della $r$ non soltanto secondo la posizione nella struttura sillabica, ma anche in base a fattori lessicali, sociali e generazionali. Dopo un'analisi fonetica molto dettagliata, che studia una moltitudine di varianti sulla scala sonora che si comportano in parte come consonanti e in parte come elementi di dittongo, l'autrice propone una spiegazione fonologica d'insieme che cerca di rendere conto dei dati raccolti mostrando la complessità delle oscillazioni tra le realizzazioni di superficie e le strutture soggiacenti.

Nella sezione «Le français en Haïti», troviamo due testi dedicati a questa varietà. Apre la sezione il contributo Le français d'Haïti (dans sa relation osmotique avec le créole): remarques à propos des sources existantes in cui Dominique FATTIER cerca di far conoscere questa varietà di francese grazie a un percorso attraverso i documenti che ne attestano le particolarità. Dopo alcune considerazioni generali sulla storia del francese in quest'isola, il concetto di ibridazione e la metafora di osmosi per descrivere la relazione tra francese regionale e creolo, sono presentati i testi antichi, il corpus del francese letterario haïtiano del xx secolo, alcuni lavori linguistici e l'atlante linguistico del creolo di Haïti. Il corpus di dati qui descritto può aiutare a conoscere la storia poco studiata e mal documentata del francese regionale delle Antille e servire da base per 
ulteriori studi sul tema. Segue l'articolo di Obrillant DAmus, De la cueillette des données empiriques en Haïti à la construction 'linguistique' de la réalité, che, partendo da un corpus lessicale di dati, studia l'inter-influenza costante tra creolo di Haïti e francese, in particolare al livello lessicale dei prestiti, dimostrando che, anche se il creolo arricchisce incessantemente il francese, il francese resta la lingua modello e di prestigio. L'autore illustra inoltre la necessità delle scienze umane di inventarsi la propria terminologia in francese per parlare della realtà culturale di Haïti, ampliando la stessa lingua francese tramite procedimenti di derivazione, composizione e prestiti.

6 L'ultima sezione, «Lexicographie», riunisce due articoli. Il primo, La nouvelle BDLPAntilles di André thiBAult, tratta della costruzione e dell'ampliamento di una sezione della Banque de Données Lexicographiques Panfrancophones (www.bdlp.org) dedicata al francese regionale delle Antille. Dopo avere illustrato gli articoli utilizzati come base per la costruzione di questa sezione della BDLP, gli autori citati nei lemmi, i campi onomasiologici rappresentati e i rinvii ad altre zone francofone, Thibault sostiene che la nuova BDLP-Antilles deve essere capace di rendere conto del fatto che le particolarità lessicali delle Antille sono rilevabili anche in altre aree francofone. L'ultimo articolo, L'étude lexicale du français régional des Antilles: à la recherche d'une méthodologie appropriée di Teodor ZANOAGA, mostra come lavorare sul lessico del francese delle Antille, il cui inserimento nella banca-dati BDLP fa sì che ad ogni passo ci si debba porre questioni metodologiche, a tutti i livelli, dalla lemmatizzazione delle entrate alla scelta delle prime attestazioni.

7 Il volume non solo illustra gli studi linguistici più recenti sul tema, ma riesce anche a dare una panoramica dei principali problemi nello studio del francese regionale delle Antille sull'asse storico, lessicale, fonetico e letterario presentandosi come un ottimo punto di partenza sia per chi si è appena avvicinato a questa varietà di francese, sia per i ricercatori già specializzati in questo ambito di ricerca. 